4. Baillat, D., Begue, A., Stehelin, D., and Aumer cier, M. 2002. ETS-1 transcription factor binds cooperatively to the palindromic head to head ETS-binding sites of the stromelysin- 1 promoter by counteracting autoinhibition. J. Biol. Chem. 277:29386-29398.

5. Iwasaka, C., Tanaka, K., Abe, M., and Sato, Y. 1996. Ets-1 regulates angiogenesis by inducing the expression of urokinase-type plasminogen activator and matrix metalloproteinase- 1 and the migration of vascular endothelial cells. J. Cell. Physiol. 169:522-531.

6. Dandre, F., and Owens, G.K. 2004. Platelet-derived growth factor-BB and Ets-1 transcription fac- tor negatively regulate transcription of multiple smooth muscle cell differentiation marker genes. Am. J. Physiol. 286:H2042-H2051.

7. Kuwahara, K., Barrientos, T., Pipes, G.C., Li, S., and Olson, E.N. 2005. Muscle-specific signaling mechanism that links actin dynamics to serum response factor. Mol. Cell. Biol. 25:3173-3181.

8. Lockman, K., et al. 2004. Sphingosine 1-phosphate stimulates smooth muscle cell differentiation and proliferation by activating separate serum response factor co-factors. J. Biol. Chem. 279:42422-42430.

9. Graiani, G., et al. 2005. Genetic deletion of the p66Shc adaptor protein protects from angiotensin II-induced myocardial damage. Hypertension.
46:433-440.

10. Vecchione, C., et al. 2005. Protection from angiotensin II-mediated vasculotoxic and hypertensive response in mice lacking PI3Kgamma. J. Exp. Med. 201:1217-1228.

11. Shindo, T., et al. 2002. Krüppel-like zinc-finger transcription factor KLF5/BTEB2 is a target for angiotensin II signaling and an essential regulator of cardiovascular remodeling. Nat. Med. 8:856-863.

12. Kohout, T.A., and Lefkowitz, R.J. 2003. Regulation of $\mathrm{G}$ protein-coupled receptor kinases and arrestins during receptor desensitization. Mol. Pharmacol. 63:9-18.

\title{
Osteoblast-derived PTHrP is a physiological regulator of bone formation
}

\author{
T. John Martin
}

St. Vincent's Institute of Medical Research and Department of Medicine, University of Melbourne, Melbourne, Victoria, Australia.

\begin{abstract}
Parathyroid hormone-related protein (PTHrP) acts as a paracrine regulator in several tissues, and its physiological roles also extend to bone. In this issue of the JCI, Miao et al. demonstrate that osteoblast-specific ablation of Pthrp in mice results in osteoporosis and impaired bone formation both in vivo and ex vivo (see the related article beginning on page 2402). These mice recapitulate the phenotype of mice with haploinsufficiency of Pthrp. The findings demonstrate that PTHrP plays a central role in the physiological regulation of bone formation, by promoting recruitment and survival of osteoblasts, and probably plays a role in the physiological regulation of bone resorption, by enhancing osteoclast formation. This has implications for both our understanding of the pathogenesis of osteoporosis and its treatment.
\end{abstract}

\section{Multiple sites of PTHrP production and action}

Parathyroid hormone-related protein (PTHrP) resembles parathyroid hormone (PTH) in its aminoterminal sequence (1), and the two have very similar structural requirements for binding and activation of their common receptor, type 1 PTH receptor (PTHR1) (2). Although PTHrP circulates as a hormone that causes hypercalcemia in patients with certain cancers, it has no normal hormonal role except in the fetus, where it promotes the transfer of calcium across the placenta from mother to fetus, and during late pregnancy and lactation, in which its function is still not fully defined $(3,4)$. Largely as a result of genetics-based studies, we now realize that PTHrP should not

Nonstandard abbreviations used: PTH, parathyroid hormone; PTHR1, type 1 PTH receptor; PTHrP, PTHrelated protein; RANK, receptor activator of NF-KB; RANKL, RANK ligand.

Conflict of interest: The author is a consultant to the Chugai Pharmaceutical Co.

Citation for this article: J. Clin. Invest. 115:2322-2324 (2005). doi:10.1172/JCI26239. be regarded as a hormone at all in its normal role. The physiological role of PTHrP in postnatal mammals appears to relate to its function as a paracrine effector. PTHrP protein and mRNA are both widely expressed in human tissues $(3,5)$, often playing important roles during both development and adulthood. Much of our understanding of the role of PTHrP has come from the analysis of genetically manipulated mice. For example, PTHrP has been shown to regulate epithelial-mesenchymal signaling during the development of mammary glands and hair follicles, as well as the relaxation of uterine and vascular smooth muscle (4). The fact that PTHrP mRNA is unstable, and that the protein itself is liable to extensive proteolytic processing, ideally equips $\mathrm{PTHrP}$ to function as a cytokine. The status of PTHrP as a paracrine or autocrine factor has been the subject of several reviews (3-6).

\section{PTHrP in bone}

The discovery of PTHrP production in bone $(7,8)$ pointed to possible local functions of this peptide, and ablation of either
Pthrp or Pthr1 in mice revealed a role for PTHrP in endochondral bone formation $(9,10)$. Targeted disruption of these genes in mice resulted in gross skeletal abnormalities consistent with chondrodysplasia, and death in the perinatal period $(9,10)$. PTHrP mRNA and protein were shown to be produced also in intramembranous bone, by cells at even the earliest mesenchymal stage of development (11). Other in vitro and in vivo observations indicated that PTHrP and PTHR1 were expressed by different cell populations. Cells of mesenchymal lineage that differentiate into osteoblasts were shown to produce PTHrP very early during differentiation, and PTHrP levels decreased with further cell maturation. PTHR1 is expressed at a later stage in differentiation, by committed preosteoblasts $(7,8)$.

\section{PTH, the hormone; PTHrP, the bone cytokine}

Some years ago Amizuka et al. showed that although thrp $^{-/-}$mice died at the time of birth because of a cartilage defect, Pthrp ${ }^{+/-}$mice survived. By 3 months of age the haplotype-insufficient mice were markedly osteoporotic and possessed an increased number of adipocytes in their bone marrow (9). These observations gave much impetus to the view that PTHrP is a local factor of some importance in bone. In order to determine the relative contributions to bone physiology of the endocrine factor PTH and the paracrine factor PTHrP, Miao et al. recently examined mice rendered null for $P$ th and found that they had increased trabecular bone volume because 


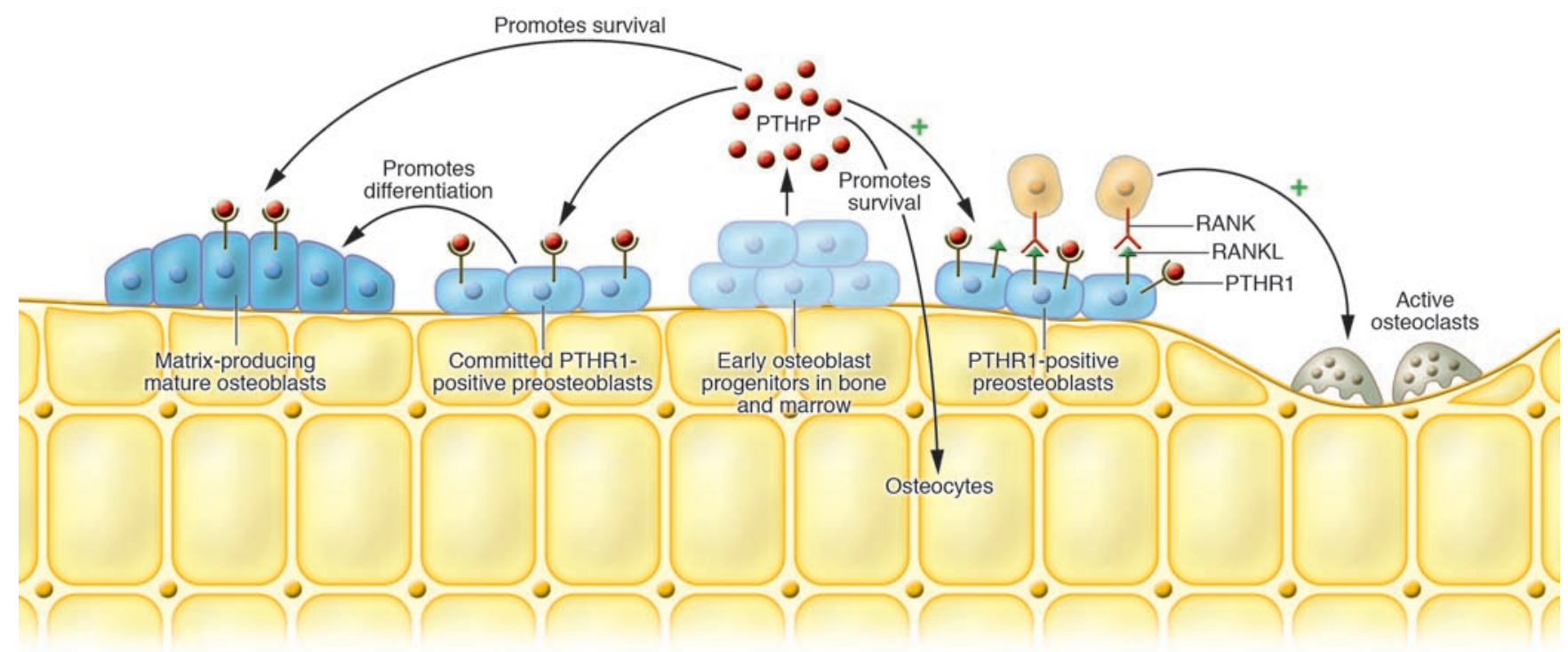

Figure 1

Paracrine actions of PTHrP in bone. Osteoblast progenitors in bone and bone marrow produce PTHrP that acts through PTHR1 on committed preosteoblasts to enhance their differentiation to mature, matrix-producing osteoblasts. The second major effect related to stimulation of bone formation is the action of PTHrP on both mature osteoblasts and osteocytes to reduce apoptosis. For PTHrP to generate an anabolic response, it needs to be presented to its targets transiently (16-18). The effect of PTHrP on the bone resorption pathway is depicted, with PTHrP acting through PTHR1 on cells of the osteoblast lineage, which respond with increased production of RANKL. This requires more prolonged stimulation by $\operatorname{PTHrP}(17,18)$, and the location of these responsive cells is such that RANKL can interact with its receptor, RANK, on hemopoietic precursors to promote increased osteoclast formation and activity. In each of its local actions the potential exists for other biological activities of PTHrP to be exerted on its target cells.

of diminished PTH-induced osteoclast formation (12). The authors went on to cross $\mathrm{Pth}^{-/-}$with $\mathrm{Pthrp}^{+/-}$mice and showed that $P t b^{-/-} P t h r p^{+/-}$mice had reduced trabecular bone volume despite the increased bone volume observed in Pth-null mice (12). The authors concluded that, postnatally, PTH is secreted as a hormone in response to a hypocalcemic signal in order to regulate calcium homeostasis by promoting bone resorption, whereas PTHrP functions as a bone cytokine to control bone mass.

\section{Osteoblast-derived PTHrP is a physiological regulator of bone turnover}

In this issue of the JCI, Miao et al. present compelling evidence supporting the notion of a paracrine role for PTHrP as a pivotal endogenous stimulator of bone formation that acts on committed osteoblast precursors in order to enhance their differentiation and, furthermore, to reduce osteoblast apoptosis (13). The authors generated mice with an osteoblast-specific targeted disruption of Pthrp using Cre-LoxP technology. These mice were shown to recapitulate the bone phenotype of $\mathrm{Pthr}^{+/-}$mice in that, even in the presence of normal levels of circulating PTH, they have marked osteo- porosis characterized by impaired bone formation, resulting from an impaired capacity to generate osteoblasts from bone marrow precursors and increased osteoblast apoptosis. The authors suggest that the absence of an increased number of adipocytes in the bone marrow of these mice is likely explained by the timing of Pthrp ablation in the mesenchymal cell differentiation process (13). Furthermore, in both the Pthrp-haploinsufficient mice described previously (9) and the osteoblast-specific Pthrp knockout mice reported in their current study (13), the number of osteoclasts was decreased in bone. Although the emphasis in the work presented in the authors' current study is on the role of PTHrP in the mechanism of osteoblast formation during bone remodeling, this important observation is also consistent with a paracrine role for PTHrP in osteoclast formation. The results of the current study establish osteoblast-derived PTHrP as a physiological regulator of bone remodeling. This is illustrated in Figure 1, which shows PTHrP being produced by cells of early osteoblast lineage that do not express PTHR1. PTHrP acts on PTHR1-positive committed preosteoblasts, and these cells respond by differentiating into mature, synthetic osteo- blasts. PTHrP also acts directly on mature osteoblasts and osteocytes to prevent their apoptosis. The evidence that ablation of Pthrp also results in reduced numbers of osteoclasts (13) is consistent with a requirement for PTHrP to enhance production of the osteoclast growth factor receptor activator of NF- $\mathrm{KB}$ ligand (RANKL) by PTHR1positive preosteoblasts (Figure 1). As a result, osteoclast formation is promoted by interaction of the membrane molecule, RANKL, with its receptor, receptor activator of NF-KB (RANK).

This genetic approach (13) has revealed PTHrP as the crucial ligand for PTHR1 in bone, with actions that reproduce the known effects of PTH, that is, the stimulation of bone formation by promotion of the differentiation of committed osteoblast precursors (14) as well as by inhibition of apoptosis of mature osteoblasts and osteocytes (15). The great therapeutic efficacy of PTH as a skeletal bone anabolic agent has been established through studies of osteoporosis (16); this efficacy is dependent on intermittent injections, each resulting in a sharp peak of PTH levels in the blood (17). More sustained elevation of PTH levels favors osteoclast formation through the generation of RANKL in target 
cells (18), which promotes osteoclast production from hemopoietic precursors. An important implication of the work of Miao et al. (13) is therefore that the local release of PTHrP from osteoblastic cells must be tightly regulated in time and space. Their finding that PTH treatment has enhanced anabolic efficacy in Pthrp-haploinsufficient mice (13) is further consistent with a pivotal role for PTHrP in bone formation. Since both osteoblast differentiation and survival are impaired in PTHrP-deficient mice, PTH treatment must first correct that deficiency, and then superimpose the anabolic response that would be expected in normal mice. The authors raise the plausible possibility that PTHrP deficiency could enhance the therapeutic response to PTH, thus identifying osteoporotic patients more likely to respond to this treatment (13). The difficulty at present would be to find ways to identify PTHrP-deficient human subjects.

\section{Implications of this newly revealed role of PTHrP}

This new insight into the role of PTHrP in bone physiology poses a number of intriguing questions. First, for PTHrP to stimulate bone formation by enhancing osteoblast differentiation and reducing osteoblast apoptosis, control mechanisms must exist to ensure that only short-lived, high levels of PTHrP are available to local targets in order to favor bone formation, as persistently increased local PTHrP levels would favor increased osteoclast formation, through stimulation of RANKL production (Figure 1). PTHrP release needs to be exquisitely regulated in terms of concentration, location, and time, so that it is presented only briefly to these target cells. On the other hand, the spatiotemporal controls might be such that excessive osteoclast formation is much less likely under these conditions in comparison with when PTH is presented systemically to the whole skeleton. We can only speculate about mechanisms that might achieve such fine control. They could involve cytokinemediated and/or neural control of PTHrP gene transcription, mRNA stability, or proteolytic processing of the protein.

Second, the molecule being presented locally to target cells is likely to be much more than simply the aminoterminal domain of PTHrP, but rather the fulllength PTHrP molecule and/or its posttranslational proteolytic products. PTHrP has been shown to contain a number of biologically active domains distinct from the aminoterminal region (reviewed in refs.
$3,5,6,19)$ and is subject to proteolytic processing at several sites (19). Could any of its constituent activities modify the physiological action of PTHrP that is mediated by its interaction with PTHR1? The efficacy of the 1-34 aminoterminal fragment of PTH (PTH 1-34) is established (16), and PTH 1-84 is in clinical trials for the treatment of osteoporosis. PTHrP 1-36 has been shown to have an anabolic effect in human subjects (20), as would be expected, but it is difficult to see how this effect would differ appreciably from that of the amino terminal of $\mathrm{PTH}$, unless perhaps by virtue of different pharmacokinetics. The possible contribution of other biologically active domains of PTHrP (19), including its nuclear translocation domain (21), could be addressed with genetic and pharmacological approaches.

Third, could some forms of osteoporosis result from inadequate PTHrP production on a genetic or acquired basis, or insufficient release? Either of these circumstances could lead to impaired bone formation, and also possibly to a modified response to therapeutic approaches based on interactions between PTHR1 and its ligands. From an evolutionary point of view, PTHrP deficiency could contribute to the age-related decrease in bone formation that takes place at the bone multicellular level, since, once bone has grown to adulthood, there is less need for continued bone-forming ability. On the other hand, excessive or prolonged local PTHrP production could contribute to osteoclast formation, increased bone resorption, and high-turnover osteoporosis. Identification of such possible states of PTHrP deficiency and excess in bone would be worth pursuing in the light of this new evidence.

The maintenance of skeletal integrity is an essential survival function for mammals. Participation in this process by PTHrP might be sufficient to explain the remarkable conservation of PTHrP amino acid sequence among mammalian species. Final$1 y$, answers to the questions raised by this work will surely influence future approaches to anabolic therapies for the skeleton.

\section{Acknowledgments}

The author acknowledges the helpful advice of Ego Seeman.

Address correspondence to: T. John Martin, St. Vincent's Institute of Medical Research, 9 Princes Street, Fitzroy, 3065 Victoria, Australia. Phone: 61-3-9288-2480; Fax: 613-9416-2676; E-mail: jmartin@svi.edu.au.
1. Suva, L.J., et al. 1987. A parathyroid hormone-related protein implicated in malignant hypercalcemia: cloning and expression. Science. 237:893-896.

2. Juppner, H., et al. 1991. A G protein-linked receptor for parathyroid hormone and parathyroid hormone-related peptide. Science. 254:1024-1026.

3. Philbrick, W.M., et al. 1996. Defining the roles of PTHrP in normal physiology. Physiol. Rev. 76:127-173.

4. Wysolmerski, J.J., Stewart, A.F., and Martin T.J. 2001. Physiological actions of PTH and PTHrP. V. Epidermal, mammary, reproductive and pancreatic tissue. In The parathyroids. 2 nd edition. J.P. Bilezikian, R.L. Marcus, and M.A. Levine, editors. Academic Press Inc. San Diego, California, USA/ London, United Kingdom. 275-291.

5. Martin, T.J., Moseley, J.M., and Williams, E.D. 1997. Parathyroid hormone-related protein: hormone and cytokine. J. Endocrinol. 154(Suppl.):S23-S37.

6. Martin, T.J., Lam, M.H.C., Kartsogiannis, V., and Gillespie, M.T. 2000. Parathyroid hormone-related protein. In Skeletal growth factors. E. Canalis, editor. Lippincott Williams \& Wilkins. Philadelphia, Pennsylvania, USA. 335-354.

7. Moseley, J.M., et al. 1991. Immunohistochemical detection of PTHrP in human fetal epithelia. J. Clin. Endocrinol. Metab. 73:478-484.

8. Suda, N., et al. 1996. Expression of parathyroid hormone-related protein in cells of osteoblast lineage. J. Cell. Physiol. 166:94-104.

9. Amizuka, N., et al. 1996. Haploinsufficiency of parathyroid hormone-related peptide (PTHrP) results in abnormal postnatal bone development. Dev. Biol. 175:166-176.

10. Lanske, B., et al. 1996. PTH/PTHrP receptor in early development and Indian hedgehog-regulated bone growth. Science. 273:663-666.

11. Kartsogiannis, V., et al. 1997. Temporal expression of PTHrP during endochondral bone formation in mouse and intramembranous bone formation in an in vivo rabbit model. Bone. 1:385-391.

12. Miao, D., et al. 2004. Parathyroid-hormone-related peptide is required for increased trabecular bone volume in parathyroid hormone-null mice. Endocrinology. 145:3554-3562.

13. Miao, D., et al. 2005. Osteoblast-derived PTHrP is a potent endogenous bone anabolic agent that modifies the therapeutic efficacy of administered PTH 1-34. J. Clin. Invest. 115:2402-2411. doi:10.1172/JCI24918.

14. Dobnig, H., and Turner, R.T. 1995. Evidence that intermittent treatment with parathyroid hormone increases bone formation in adult rats by activation of bone lining cells. Endocrinology. 136:3632-3638.

15. Jilka, R.L., et al. 1999. Increased bone formation by prevention of osteoblast apoptosis with parathyroid hormone. J. Clin. Invest. 104:439-446.

16. Neer, R.M., et al. 2001. Effect of parathyroid hormone (1-34) on fractures and bone mineral density in post-menopausal women with osteoporosis. N. Engl. J. Med. 344:1434-1441.

17. Frolik, C.A., et al. 2003. Anabolic and catabolic bone effects of human parathyroid hormone (1-34) are predicted by duration of hormone exposure. Bone. 33:372-379.

18. Ma, Y.L., et al. 2001. Catabolic effects of continuous human PTH (1-38) in vivo is associated with sustained stimulation of RANKL and inhibition of osteoprotegerin and gene-associated bone formation. Endocrinology. 142:4047-4054.

19. Orloff, J.J., et al. 1994. Parathyroid hormone-related protein as a prohormone: posttranslational processing and receptor interactions. Endocr. Rev. 15:40-60.

20. Horwitz, M.J., et al. 2003. Short term, high-dose PTHrP as a skeletal anabolic agent for the treatment of postmenopausal osteoporosis. J. Clin. Endocrinol. Metab. 88:569-575.

21. Lam, M.H., et al. 1999. Importin beta recognizes parathyroid hormone-related protein with high affinity and mediates its nuclear import in the absence of importin alpha. J. Biol. Chem. 274:7391-7398. 